\title{
New Approach to Enrich Pasta with Polyphenols from Grape Marc
}

\author{
V. Marinelli, L. Padalino, D. Nardiello, M. A. Del Nobile, and A. Conte \\ Services Center of Applied Research, University of Foggia, Via Napoli 25, 71122 Foggia, Italy \\ Correspondence should be addressed to A. Conte; amalia.conte@unifg.it
}

Received 7 September 2015; Revised 27 October 2015; Accepted 28 October 2015

Academic Editor: Somdet Srichairatanakool

Copyright (c) 2015 V. Marinelli et al. This is an open access article distributed under the Creative Commons Attribution License, which permits unrestricted use, distribution, and reproduction in any medium, provided the original work is properly cited.

\begin{abstract}
Food industry produces significant amount of waste that represents a problem for the sector. However, by-products are also promising sources of compounds which may be reused for their nutritional properties. The aim of this work is to exploit winemaking by-products, obtaining an extract by ultrasound-assisted extraction only using water as solvent. The characteristics of spaghetti enriched with grape marc were assessed and compared to control samples. In particular, total phenolic and flavonoids contents, the antioxidant activity, the cooking quality, and the sensory acceptability were evaluated at various steps of pasta production. The enriched spaghetti showed higher total phenolic and flavonoids contents and higher antioxidant activity than the control pasta. In addition, low cooking losses were found. In terms of sensory properties fortified pasta is acceptable as the traditional product, thus demonstrating that it is possible to exploit food waste to better satisfy consumer demand for healthy food products in a more sustainable perspective.
\end{abstract}

\section{Introduction}

Food industry and in particular fruit and vegetable processing generally produce significant wastes that represent the major disposal problem for food sector, from both the environmental and the economic point of view. However, it is worth noting that by-products can be also promising sources of compounds, being rich in carotenoids, polyphenols, tocopherols, vitamins, and other substances [1]. In general, agroindustrial wastes are disposed, utilized as animal feed or as fertilizer, but their use as sources of natural food ingredients recently recorded considerable attention from food research. The interest for by-products is due to the fact that they represent raw materials at low cost and are widely available. In addition, the natural compounds contained in by-products could allow satisfying consumer demand for food products with new functional properties, typical of compounds from plant world, which are natural and health-promoting. It is widely recognized that an adequate consumption of fruit and vegetables plays an important role in the prevention of diseases, for example, reduced risk of heart disease and stroke, as well as certain types of cancer [1]. Due to this consumer demand for healthier foods and due to the environmental consequences of by-products, food industry points towards formulation of products enriched with bioactive compounds that can be also extracted from by-products [2]. In the scientific literature several extraction techniques have been proposed. Some of them are traditional systems based on the extraction power of solvents or heat application and other ones are unconventional techniques of new generation as the ultrasound-assisted extraction (UAE). This extraction technique is preferred compared to the classical extraction methods because it allows to shorten the extraction time, reduces the organic solvent waste, increases the extraction yield, and enhances the quality of extracts and it is possible to use water as solvent $[3,4]$. The UAE is based on sound mechanical waves that go beyond human hearing, whose frequencies are superior to $20 \mathrm{kHz}$. This type of extraction requires a liquid medium previously selected that allows ultrasonic wave to propagate up to the product, also preserving the integrity of the molecules that can be thermolabile, thermostable, hydrosoluble, and liposoluble. This is possible due to the cavitation forces resulting from the ultrasound application. This phenomenon could be explained as the formation and the final collapse of microbubbles 
into the liquid medium that may cause different mechanical effects, such as turbulent streaming, particle collisions, and cell wall disruption. These mechanical effects could cause a greater penetration of the solvent into the food matrix and, consequently, an increase of the mass transfer rates of the bioactive compounds from the food matrix into the extraction solvent [3]. Under these conditions the transfer of compounds to be extracted is facilitated and the extraction time is reduced.

According to the literature consulted, most of the studies on by-products are focused on optimization conditions for the extraction to obtain potential bioactive compounds, but the applications of the extracted substances to foods are very scarce. Calvo et al. [5] have designed sausages formulation enriched with lycopene by means of direct addition of tomato skins, previously dried and ground. The same group of researchers also studied the influence on the physicochemical and sensorial properties of raw and cooked hamburgers enriched with dry tomato peels, demonstrating that the addition of tomato peel to meat products results in healthier product due to both lycopene and fiber content present in tomato by-product [6]. Özvural and Vural [7] incorporated grape seed flour, rich in polyphenols obtained from wine byproducts, into frankfurters.

In this context, grape marc is taken into account, being an interesting source of natural compounds as polyphenols (anthocyanins, catechins, flavonols, and phenolic acids) and fibers [3]. The aim of this work was to enrich fresh and dry pasta with grape marc extracts obtained by means of UAE using only water as solvent extraction, thus demonstrating that it is possible to increase polyphenols amount and antioxidant activity of pasta without compromising the sensory characteristics and valorize wine-making by-products that are generally discarded.

\section{Material and Methods}

2.1. Raw Materials. Grape marc made up of skins, seeds, and stalks was provided by a local company of Foggia (Southern Italy), during the 2014 harvest. The samples were dried at 30$35^{\circ} \mathrm{C}$ in a dryer (SG600, Namad, Rome, Italy) for 48 hours. The dried grape marc was reduced in a fine powder by a hammer mill (16/BV-Beccaria s.r.l., Cuneo, Italy) and then stored at $4^{\circ} \mathrm{C}$ until further utilization.

2.2. Chemicals. Folin-Ciocalteu reagent, gallic acid monohydrate, methanol, hydrochloric acid, ABTS (2,2-azino-bis(3ethylbenzothiazoline-6-sulfonic acid diammonium salt), potassium persulfate $\left(\mathrm{K}_{2} \mathrm{~S}_{2} \mathrm{O}_{8}\right)$, Trolox (6-hydroxy-2,5,7,8tetramethylchroman-2-carboxylic acid), aluminium chloride $\left(\mathrm{AlCl}_{3}\right)$, sodium nitrite $\left(\mathrm{NaNO}_{2}\right)$, sodium hydroxide solution $(\mathrm{NaOH})$, and quercetin were supplied from Sigma-Aldrich (Milan, Italy). Anhydrous sodium carbonate $\left(\mathrm{Na}_{2} \mathrm{CO}_{3}\right)$ was supplied from Carlo Erba (Milan, Italy). For the preparation of the phosphate buffered saline (PBS), the following salts were used: sodium phosphate dibasic heptahydrate $\left(\mathrm{HNa}_{2} \mathrm{O}_{4} \mathrm{P} \cdot 7 \mathrm{H}_{2} \mathrm{O}\right)$ and sodium phosphate monobasic monohydrate $\left(\mathrm{H}_{2} \mathrm{NaO}_{4} \mathrm{P} \cdot \mathrm{H}_{2} \mathrm{O}\right)$. These were purchased from
Sigma-Aldrich (Milan, Italy). All reagents were of analytical grade.

2.3. Extraction Process. Grape marc extract (GME) was obtained by means of UAE (USR-1500-50WL, Weal s.r.l., Milan, Italy) using only water as solvent. In the reactor grape marc was suspended in water at a ratio of $1: 10(\mathrm{w} / \mathrm{v})$ and ultrasonically treated for 60 minutes at acoustic frequency of $25 \mathrm{kHz}$ and with ultrasonic power density of $50 \mathrm{~W} / \mathrm{L}$. The obtained extract was centrifuged at $10000 \mathrm{rpm}$ for 10 minutes at room temperature and then it was filtered by means of $0.45 \mu \mathrm{m}$ PTFE filters (Teknokroma, Sant Cugat del Vallés, Barcelona, Spain) and stored at $-20^{\circ} \mathrm{C}$ until the analytical determinations.

2.4. Total Phenols, Flavonoids, and Antioxidant Activity Determination. To determine total phenols, flavonoids, and antioxidant activity, the extraction was performed as described by Biney and Beta [8] from all the various samples: dough, extruded fresh spaghetti, pasteurized fresh spaghetti, dry spaghetti, and cooked fresh and cooked dry spaghetti. The samples were dried at $30^{\circ} \mathrm{C}$, grounded, and sieved through an $800 \mu \mathrm{m}$ sieve. For total phenols and antioxidant activity determination, $2 \mathrm{~g}$ of powdered sample was mixed with $20 \mathrm{~mL}$ of acidified methanol $\left(\mathrm{HCl} / \mathrm{H}_{2} \mathrm{O}: \mathrm{MeOH}\right.$, $20: 80$ ), while, in the case of flavonoids, the same quantity of sample was combined with $10 \mathrm{~mL}$ of acidified methanol. The mixtures were included in $50 \mathrm{~mL}$ centrifuge tubes and shaken at room temperature in darkness for $2 \mathrm{~h}$ at $300 \mathrm{rpm}$ using orbital shaker (HS 260 BASIC, IKA, Staufen, Germany). Next, the samples were centrifuged at $5^{\circ} \mathrm{C}$ for 15 minutes at $10000 \mathrm{rpm}$ (5804R, Eppendorf, Milan, Italy) and supernatant was collected and filtered (PTFE, $0.45 \mu \mathrm{m}$ ) prior to the analytical determinations.

2.5. Spaghetti Preparation. Commercial durum wheat semolina was purchased from Agostini Mill (Montefiore dell'Aso, Italy). Semolina was mixed with proper amount of water or grape marc extract (GME) in the rotary shaft mixer (Namad, Rome, Italy) at $25^{\circ} \mathrm{C}$ for $20 \mathrm{~min}$ to distribute liquid uniformly throughout the semolina particles. The dough was extruded with a 60VR extruder (Namad) as described by Padalino et al. [9]. After extrusion, pasta was pasteurized with steam for $3 \mathrm{~min}$ at $90^{\circ} \mathrm{C}$ (Namad, Rome, Italy). The pasteurization system uses air at room temperature to cool down the pasta after the thermal treatment. Then, the extruded pasta was dried in a dryer (SG600; Namad). The drying process conditions applied were in accordance with Padalino et al. [9].

2.6. Sensory Analysis. Fresh-extruded and dry spaghetti samples were submitted to a panel of 15 trained tasters (seven men and eight women, aged between 28 and 45 years) in order to evaluate the sensory attributes. The panelists were selected on the basis of their sensory skills (ability to accurately determine and communicate the sensory attributes as appearance, odor, flavor, and texture of a product). The panelists were also trained in sensory vocabulary and identification of 
particular attributes by evaluating durum wheat commercial spaghetti [10]. They were asked to indicate color, homogeneity, and resistance to breaking of fresh and dry uncooked spaghetti. In addition, to color and homogeneity, odor was also evaluated for fresh-extruded and pasteurized spaghetti. Elasticity, firmness, bulkiness, adhesiveness, color, odor, and taste were evaluated on fresh and dry cooked spaghetti. To the aim, a nine-point scale, where 1 corresponded to extremely unpleasant, 9 to extremely pleasant, and 5 to the threshold acceptability, was used to quantify each attribute. On the basis of the abovementioned attributes, panelists were also asked to score the overall quality of both cooked and uncooked products using the same nine-point scale [9].

2.7. Cooking Quality. The optimal cooking time (OCT) was evaluated according to the AACC [11] approved method 6650. The cooking loss, that is, the amount of solid substance lost into the cooking water, was determined according to the AACC [11] approved method 66-50. The swelling index and the water absorption of cooked pasta (grams of water per gram of dry pasta) were determined according to the procedure described by Padalino et al. [12]. Moreover, cooked spaghetti samples were submitted to hardness and adhesiveness analysis by means of a Zwick/Roell model Z010 Texture Analyzer (Zwick Roell Italia s.r.l., Genoa, Italy) equipped with a stainless steel cylinder probe ( $2 \mathrm{~cm}$ diameter). The hardness (mean maximum force, $\mathrm{N}$ ) and adhesiveness (mean negative area, Nmm) were measured according to the procedure described by Padalino et al. [12]. Six measurements for each spaghetti sample were performed.

\subsection{Chemical Analysis}

2.8.1. Determination of Total Phenolic Compounds. Total phenolic compounds were determined by UV-vis spectrophotometry according to Folin-Ciocalteu method [13]. In particular, GME was 1:10 diluted with water before analysis, while extracts obtained from samples relative to each production step of spaghetti, previously described, were analyzed without any dilution. Specifically, $0.5 \mathrm{~mL}$ of grape marc or pasta extract was mixed with $2.5 \mathrm{~mL}$ of Folin-Ciocalteu reagent (diluted 1:10 with water) and, after 5 minutes, $2 \mathrm{~mL}$ of $\mathrm{Na}_{2} \mathrm{CO}_{3}(75 \mathrm{~g} / \mathrm{L})$ was added. The sample was kept in darkness at room temperature for 2 hours. Distilled water was used as control sample. The absorbance was measured at $765 \mathrm{~nm}$ by an UV-vis spectrophotometer (UV1800, Shimadzu Italia s.r.l., Milan, Italy). Total phenolic compounds were quantified by a calibration curve previously built $\left(3-200 \mathrm{mg} / \mathrm{L} ; R^{2}=0,9989\right)$ using standard solution of gallic acid, and the total phenolic content was expressed as $\mathrm{mg}$ gallic acid/100 g of dry weight $(\mathrm{dw})$. All tests were carried out in triplicate.

2.8.2. Determination of Antioxidant Activity. The antioxidant activity was assessed using ABTS test, which is based on the ability of antioxidants to interact with the radical cation $2,2^{\prime}$ azino-bis(3-ethylbenzothiazoline-6-sulfonic acid) $\left(\mathrm{ABTS}^{{ }^{+}}\right.$) inhibiting its absorption at $734 \mathrm{~nm}$, according to the method of Re et al. [14]. $7 \mathrm{mM}$ ABTS stock solution and $140 \mathrm{mM}$ potassium persulfate were utilized. The ABTS radical cation $\left(\mathrm{ABTS}^{\bullet+}\right.$ ) was obtained by reacting ABTS stock solution with $2.45 \mathrm{mM}$ potassium persulfate (final concentration) and allowing the mixture to stand in the dark at room temperature for $12-16 \mathrm{~h}$. The $\mathrm{ABTS}^{\bullet+}$ solution was diluted with $5 \mathrm{mM}$ phosphate buffered saline, $\mathrm{pH} 7.4$ (PBS), and absorbance 0.70 $\pm 0,02$ at $734 \mathrm{~nm}$. Then, $200 \mu \mathrm{L}$ of sample extract was added to $2 \mathrm{~mL}$ of $\mathrm{ABTS}^{\bullet+}$ diluted solution and after 3 minutes at $30^{\circ} \mathrm{C}$ the mixture was measured through a spectrophotometer (UV1800, Shimadzu Italia s.r.l., Milan, Italy) at $734 \mathrm{~nm}$. A calibration curve was previously built using 6-hydroxy-2,5,7,8tetramethylchroman-2-carboxylic acid (Trolox) as standard, at concentrations between 0.98 and $250 \mu \mathrm{M}\left(R^{2}=0,9997\right)$ and the antioxidant activity was expressed as $\mu$ moli Trolox equivalents for gram of dry weight $(\mathrm{dw})$. All analyses were carried out in triplicate.

2.8.3. Determination of Total Flavonoids. Total flavonoids content both in GME and in all the extracts was determined by aluminum chloride colorimetric method, according to Huang and Ho [15] with modifications, using quercetin as standard. Extracts $(0.5 \mathrm{~mL})$, prepared as previously described, were mixed with $2 \mathrm{~mL}$ of distilled water and $150 \mu \mathrm{L}$ of a $5 \%$ sodium nitrite $\left(\mathrm{NaNO}_{2}\right)$ solution. After 6 minutes, $150 \mu \mathrm{L}$ of a $10 \%$ aluminum chloride $\left(\mathrm{AlCl}_{3}\right)$ solution was added and the mixture was allowed to stand for 6 minutes. Finally, $1 \mathrm{~mL}$ of $1 \mathrm{M}$ sodium hydroxide $(\mathrm{NaOH})$ was added and total volume was made up to $5 \mathrm{~mL}$ with distilled water. Then, the solutions were mixed and for each sample the absorbance was read in triplicate against blank at $415 \mathrm{~nm}$. The standard curve was prepared using quercetin as standard in the range $3,13-500 \mathrm{mg} / \mathrm{L}\left(R^{2}=0,9981\right)$ and total amount of flavonoids was expressed in mg of quercetin/100 $\mathrm{g}$ of dry weight $(\mathrm{dw})$.

2.9. Statistical Analysis. Experimental data were compared by a one-way analysis of variance (ANOVA). Duncan's multiple range test, with the option of homogeneous groups $(P<$ $0.05)$, was carried out to determine significant differences between spaghetti samples. STATISTICA 7.1 for Windows (StatSoft, Inc., Tulsa, OK, USA) was used.

\section{Results and Discussion}

In this study the total phenolic and flavonoids content together with the antioxidant activity were evaluated on both control and enriched samples: dough, extruded fresh pasta, and fresh-pasteurized/dry pasta (uncooked and cooked). Details on chemical characterization, cooking quality (only for dry pasta), and sensory properties are reported below separately.

3.1. Chemical Quality. Total phenolic (mg gallic acid/ $100 \mathrm{~g} \mathrm{dw}$ ), flavonoids (mg quercetin/100 g dw), and antioxidant activity ( $\mu$ moli Trolox/g dw), measured by ABTS assay, of GME were shown in Table 1 . The obtained results indicate that the extract has a high content of polyphenols, equal 
TABLE 1: Total phenols, total flavonoids, and antioxidant activity of the grape marc extract.

\begin{tabular}{lccc}
\hline Sample & $\begin{array}{c}\text { Total phenols } \\
(\mathrm{mg} \text { gallic acid/100 g dw }) \pm \mathrm{SD}\end{array}$ & $\begin{array}{c}\text { Total flavonoids } \\
(\mathrm{mg} \text { quercetin/100 g dw }) \pm \mathrm{SD}\end{array}$ & $\begin{array}{c}\text { Antioxidant activity } \\
(\mu \mathrm{moli} \text { Trolox/g dw }) \pm \mathrm{SD}\end{array}$ \\
\hline GME & $443 \pm 1.7$ & $405 \pm 12$ & $518 \pm 7.4$ \\
\hline
\end{tabular}

GME: grape marc extract.

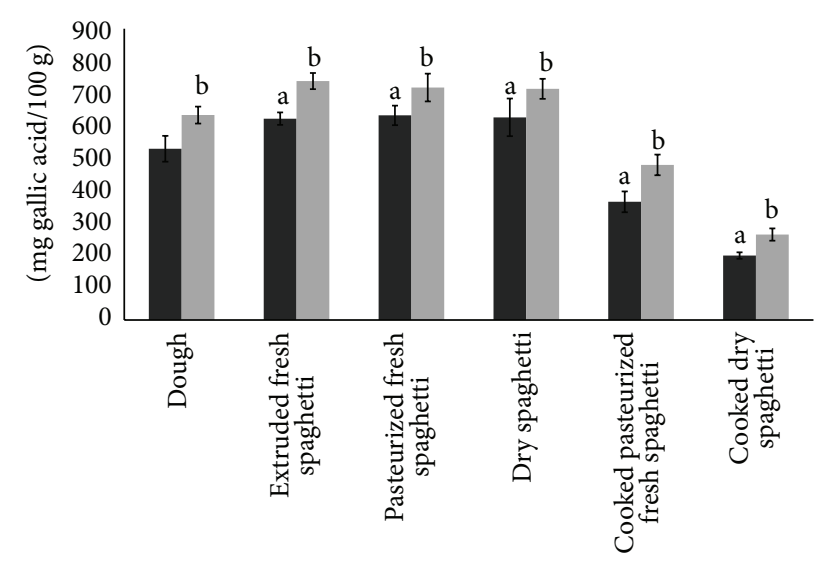

- CNT

- ACTIVE

FIgURE 1: Total phenolic content of spaghetti samples in each step of the production process. Results are expressed as means \pm SD for $n=3{ }^{\mathrm{a}, \mathrm{b}}$ Data in columns with different superscripts are significantly different $(P<0.05)$. CNT: samples without the addition of grape marc extract; ACTIVE: samples with the addition of grape marc extract.

to $443 \mathrm{mg}$ acid gallic/100 $\mathrm{g} \mathrm{dw}$, greater than that reported by González-Centeno et al. [3], who studied the effect of acoustic frequency, ultrasonic power density, and extraction time of grape pomace by UAE. Moreover, the extract was characterized by a high flavonoid content and antioxidant activity, $405 \mathrm{mg}$ quercetin/100 $\mathrm{g} \mathrm{dw}$, and $518 \mu$ moli Trolox/g of $\mathrm{dw}$, respectively.

The phenolic compounds of the experimental samples were expressed as mg gallic acid for $100 \mathrm{~g} \mathrm{dw}$ and shown in Figure 1. In every step of the spaghetti production process, the phenolic content of the ACTIVE samples was higher than spaghetti without any extract. In particular, among the experimental samples, the extruded fresh pasta showed the highest quantity of bioactive compounds ( $737 \mathrm{mg}$ gallic $\mathrm{acid} / 100 \mathrm{~g} \mathrm{dw})$. As it can be inferred by the figure, the phenolic content decreased as temperature increased; in fact the cooked dry pasta revealed the lowest phenolic value, probably due to the combination of drying and cooking procedure. The same trend was observed by Pasqualone et al. [2], who studied biscuits enriched with grape marc extract. According to Abdel-Aal and Rabalski [16] the effect of cooking on phenols is not always the same but depends on the type of bioactive compound and type of product.

ABTS assay was used to evaluate the antioxidant activity. The results are shown in Figure 2 and expressed as $\mu$ moli Trolox per gram of dry weight. According to obtained data, the fortification of spaghetti with aqueous grape marc extract

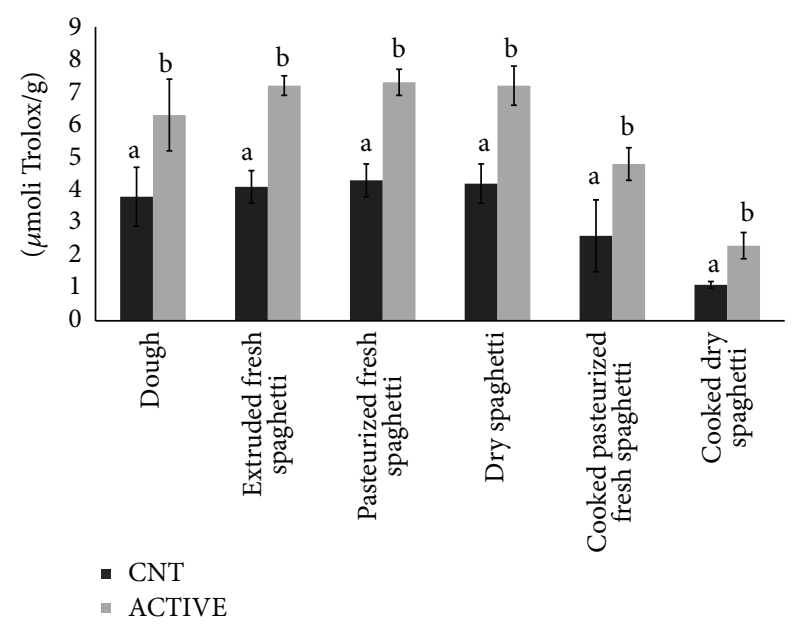

FIgURE 2: Antioxidant activity of spaghetti samples in each step of the production process. Results are expressed as means \pm SD for $n=3{ }^{\mathrm{a}, \mathrm{b}}$ Data in columns with different superscripts are significantly different $(P<0.05)$. CNT: samples without the addition of grape marc extract; ACTIVE: samples with the addition of grape marc extract.

led to a significant increase in antioxidant capacity. As in the case of total phenols, also in this case, the ACTIVE samples proved an antioxidant activity greater than the corresponding CNT sample. In fact, according to Alonso et al. [17] there is a positive correlation between the antioxidant activity and the total polyphenolic content of samples. In every step of production process a statistically significant difference between CNT and ACTIVE samples was detected. According to our findings, the entire pasta process did not cause any significant change in antioxidant activity; in fact, after extrusion an increase of antioxidant activity was observed, which remains constant in the subsequent steps. Only after cooking a decrease of antioxidant activity was found, probably because heat degraded phenols, thus decreasing their concentration.

The flavonoid content of both uncooked and cooked spaghetti is presented in Figure 3. The addition of grape marc extract to spaghetti significantly increased the flavonoid content in every phase of the production process. The highest flavonoids content was recorded for the extruded fresh pasta, which presented $58 \mathrm{mg}$ quercetin/100 g dw, greater than the dough sample enriched with the extract. According to the obtained results, it is possible to infer that the extrusion phase increased the flavonoids amount, as observed also in the case of total phenols and antioxidant activity, probably because this step frees the bounded compounds. The pasteurization and the drying process exerted little effect on flavonoids content; in fact, these phases recorded 56 and 
TABLE 2: Cooking quality of dry spaghetti samples.

\begin{tabular}{lcccccc}
\hline Sample & OCT (min) & Cooking loss (\%) & Swelling index & $\begin{array}{c}\text { Water } \\
\text { absorption (\%) }\end{array}$ & Hardness (N) & Adhesiveness (Nmm) \\
\hline CNT & 10.00 & $6.47 \pm 0.04^{\mathrm{a}}$ & $2.06 \pm 0.08^{\mathrm{a}}$ & $165 \pm 0.51^{\mathrm{a}}$ & $7.32 \pm 0.52^{\mathrm{a}}$ & $1.04 \pm 0.08^{\mathrm{a}}$ \\
ACTIVE & 10.00 & $4.53 \pm 0.21^{\mathrm{b}}$ & $1.73 \pm 0.013^{\mathrm{b}}$ & $135 \pm 0.51^{\mathrm{b}}$ & $7.25 \pm 0.56^{\mathrm{a}}$ & $0.84 \pm 0.05^{\mathrm{b}}$ \\
\hline
\end{tabular}

CNT: samples without the addition of grape marc extract.

ACTIVE: samples with the addition of grape marc extract.

${ }^{\mathrm{a}, \mathrm{b}}$ Data in columns with different superscripts are significantly different $(P<0.05)$.

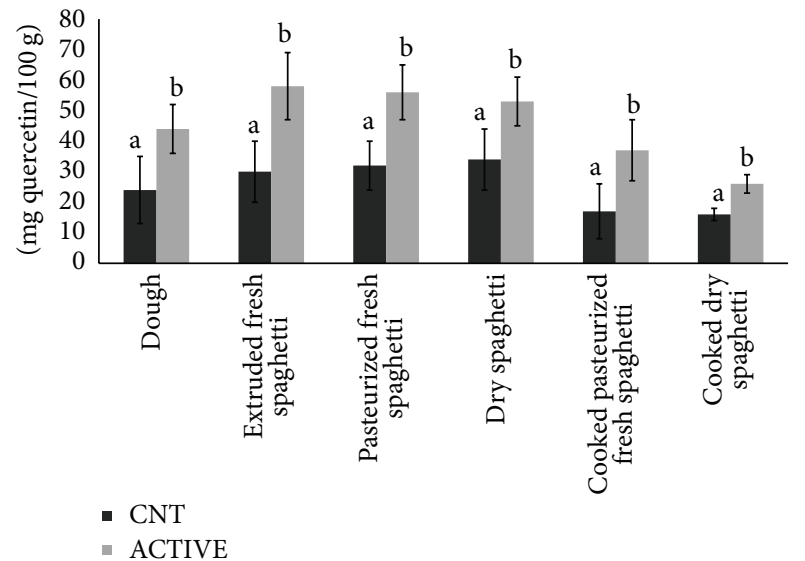

FIGURE 3: Total flavonoids of spaghetti samples in each step of the production process. Results are expressed as means \pm SD for $n=$ 3. ${ }^{\mathrm{a}, \mathrm{b}}$ Data in columns with different superscripts are significantly different $(P<0.05)$. CNT: samples without the addition of grape marc extract; ACTIVE: samples with the addition of grape marc extract.

$53 \mathrm{mg}$ quercetin $/ 100 \mathrm{~g} \mathrm{dw}$, respectively. Only after cooking a significant decrease was observed, especially in the cooked dry spaghetti.

3.2. Cooking Quality. Cooking quality is an important parameter for dry pasta evaluation. The cooking performances of the investigated spaghetti samples in terms of optimum cooking time, cooking loss, water absorption, swelling index, hardness, and adhesiveness are shown in Table 2. As can be observed from Table 2 there was no difference in optimum cooking time. In fact, for both samples studied, the optimum cooking time was around $10 \mathrm{~min}$.

The spaghetti sample with grape marc extract showed a lower cooking loss than the CNT sample. One possible explanation could be due to the capacity of the antioxidant compounds from grape marc extract present in the form of complex with proteins around the starch granules, encapsulating them during cooking and restricting excessive swelling and diffusion of the amylose content [18]. Also Rizk et al. [18] found a reduction of cooking loss in sample enriched with antioxidant compounds from tomato peels (carotenoids) with respect to the control sample (100\% wheat flour).

Regarding the water absorption and swelling index, ACTIVE sample recorded the lowest values with respect to CNT sample (Table 2). These results are in agreement with Rizk and Tolba [19] who also observed that pasta enriched with carotenoids from tomato peels had similar swelling index value of control sample (100\% wheat flour).

As compared to the CNT sample, ACTIVE sample recorded lower adhesiveness value. This could be due to the fact that the antioxidant compounds generally form with the gluten proteins a stronger gluten network that entraps the starch granules, slowing down the amylose release during cooking. On the contrary, the hardness value of ACTIVE sample was similar to that of the CNT sample.

3.3. Sensory Quality. The sensory properties of the investigated samples were evaluated by means of a group of trained panelists and the results are listed in Tables 3 and 4 for freshextruded/pasteurized and dry spaghetti samples (uncooked and cooked), respectively. Sensory data of uncooked freshextruded/pasteurized spaghetti samples (E-ACTIVE and PACTIVE) showed that addition of grape marc extract determined a slight decrease in overall quality as compared to the CNT, even though no statistically significant differences were observed among samples. Specifically, samples E-ACTIVE and P-ACTIVE recorded the smallest color scores with respect to the CNT samples. In fact, the spaghetti samples containing GME showed a light brown color in comparison to the bright yellow color of the CNT sample. Pasta color is essential for assessing pasta quality. Generally, consumers prefer pasta with a bright yellow color [20]. Moreover, the lowest color score has been observed for both pasteurized samples P-CNT and P-ACTIVE with respect to extruded samples. Most probably, the high temperature during the pasteurization temperature promoted development of Maillard reaction, giving to pasta a brownish color that slightly affects panelist judgment. Regarding the extruded and the pasteurized cooked spaghetti samples, the incorporation of GME caused a little rise of overall quality, even though no significant difference was observed between the studied samples. In particular, E-ACTIVE and P-ACTIVE spaghetti samples recorded a decline in adhesiveness and bulkiness (high score) in comparison with the E-CNT and P-CNT. Besides, the addition of GME did not determined significant differences in the other sensorial attributes. Concerning dry spaghetti (uncooked and cooked) the addition of the GME did not cause any significant differences in overall quality with respect to the CNT sample (Table 4).

\section{Conclusions}

The reutilization of wine-making by-products was proposed with success to enrich fresh or dry pasta from durum wheat. 


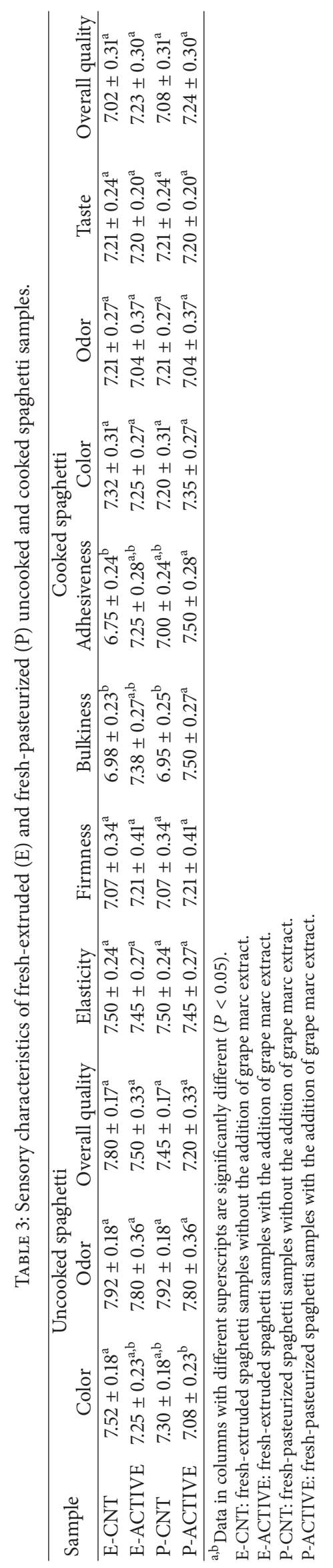




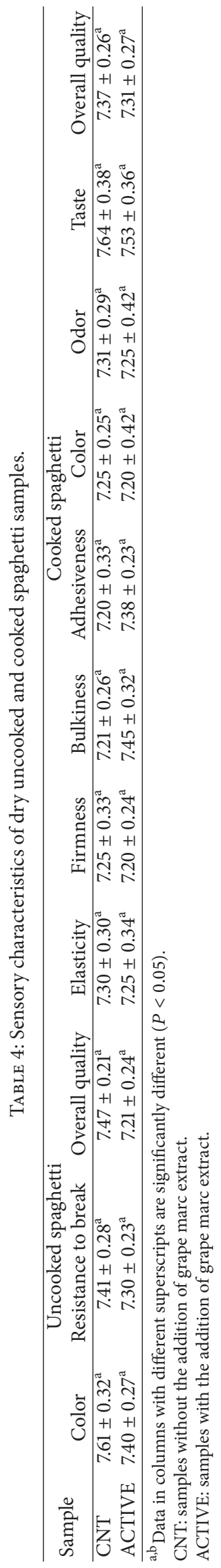


The obtained results show that it is possible to use grape marc aqueous extract, obtained from ultrasound extraction, instead of simple water to produce pasta, without altering its sensory characteristics. In fact, according to sensory analysis no significant differences have been found among the experimental samples. Moreover, enriched spaghetti was characterized by a higher content of phenolic compounds, flavonoids, and consequently antioxidant activity compared to the control sample. Therefore, data demonstrated with a concrete example that it is possible to reuse an agroindustrial waste such as grape marc to design new foods with healthful properties. In this way, it is possible to face environmental problems and satisfy at the same time consumer demand for food products with a recognized premium quality.

\section{Abbreviations}

ABTS: 2,2-Azino-bis(3-ethylbenzothiazoline)-6sulfonic acid diammonium salt

Trolox: 6-Hydroxy-2,5,7,8-tetramethylchroman-2carboxylic acid

PBS: $\quad$ Phosphate buffered saline

GME: Grape marc extract

CNT: Samples without the addition of grape marc extract

ACTIVE: Samples with the addition of grape marc extract.

\section{Conflict of Interests}

The authors declare that there is no conflict of interests regarding the publication of this paper.

\section{References}

[1] A. Schieber, F. C. Stintzing, and R. Carle, "By-products of plant food processing as a source of functional compounds-recent developments," Trends in Food Science and Technology, vol. 12, no. 11, pp. 401-413, 2001.

[2] A. Pasqualone, A. M. Bianco, V. M. Paradiso, C. Summo, G. Gambacorta, and F. Caponio, "Physico-chemical, sensory and volatile profiles of biscuits enriched with grape marc extract," Food Research International, vol. 65, pp. 385-393, 2014.

[3] M. R. González-Centeno, K. Knoerzer, H. Sabarez, S. Simal, C. Rosselló, and A. Femenia, "Effect of acoustic frequency and power density on the aqueous ultrasonic-assisted extraction of grape pomace (Vitis vinifera L.) - a response surface approach," Ultrasonics Sonochemistry, vol. 21, no. 6, pp. 2176-2184, 2014.

[4] Y. Tao, Z. Zhang, and D.-W. Sun, "Kinetic modeling of ultrasound-assisted extraction of phenolic compounds from grape marc: influence of acoustic energy density and temperature," Ultrasonics Sonochemistry, vol. 21, no. 4, pp. 1461-1469, 2014.

[5] M. M. Calvo, M. L. García, and M. D. Selgas, "Dry fermented sausages enriched with lycopene from tomato peel," Meat Science, vol. 80, no. 2, pp. 167-172, 2008.

[6] M. L. García, M. M. Calvo, and M. D. Selgas, "Beef hamburgers enriched in lycopene using dry tomato peel as an ingredient," Meat Science, vol. 83, no. 1, pp. 45-49, 2009.
[7] E. B. Özvural and H. Vural, "Grape seed flour is a viable ingredient to improve the nutritional profile and reduce lipid oxidation of frankfurters," Meat Science, vol. 88, no. 1, pp. 179183, 2011.

[8] K. Biney and T. Beta, "Phenolic profile and carbohydrate digestibility of durum spaghetti enriched with buckwheat flour and bran," LWT_Food Science and Technology, vol. 57, no. 2, pp. 569-579, 2014

[9] L. Padalino, M. Mastromatteo, L. Lecce, S. Spinelli, F. Contò, and M. A. Del Nobile, "Chemical composition, sensory and cooking quality evaluation of durum wheat spaghetti enriched with pea flour," International Journal of Food Science and Technology, vol. 49, no. 6, pp. 1544-1556, 2014.

[10] ISO Standards, "Alimentary pasta produced from durum wheat semolina-estimation of cooking quality by sensory analysispart 2: routine method," ISO 11036/7304-2:2008, 2008.

[11] AACC. American Association of Cereal Chemists, "Approved methods of the American Association of Cereal Chemistry," Method 66-50, AACC, Saint Paul, Minn, USA, 2000.

[12] L. Padalino, M. Mastromatteo, L. Lecce, S. Spinelli, F. Contò, and M. A. Del Nobile, "Chemical composition, sensory and cooking quality evaluation of durum wheat spaghetti enriched with pea flour," International Journal of Food Science \& Technology, vol. 49, no. 6, pp. 1544-1556, 2014.

[13] S. Spinelli, A. Conte, L. Lecce, A. L. Incoronato, and M. A. Del Nobile, "Microencapsulated propolis to enhance the antioxidant properties of fresh fish burgers," Journal of Food Process Engineering, vol. 38, no. 6, pp. 527-535, 2015.

[14] R. Re, N. Pellegrini, A. Proteggente, A. Pannala, M. Yang, and C. Rice-Evans, "Antioxidant activity applying an improved ABTS radical cation decolorization assay," Free Radical Biology and Medicine, vol. 26, no. 9-10, pp. 1231-1237, 1999.

[15] Y.-S. Huang and S.-C. Ho, "Polymethoxy flavones are responsible for the anti-inflammatory activity of citrus fruit peel," Food Chemistry, vol. 119, no. 3, pp. 868-873, 2010.

[16] E.-S. M. Abdel-Aal and I. Rabalski, "Effect of baking on free and bound phenolic acids in wholegrain bakery products," Journal of Cereal Science, vol. 57, no. 3, pp. 312-318, 2013.

[17] Á. M. Alonso, D. A. Guillén, C. G. Barroso, B. Puertas, and A. García, "Determination of antioxidant activity of wine byproducts and its correlation with polyphenolic content," Journal of Agricultural and Food Chemistry, vol. 50, no. 21, pp. 5832-5836, 2002.

[18] E. M. Rizk, S. H. Bedier, and M. A. Elgendy, "Utilization of carotenoid pigments extracted from tomato peel as natural antioxidants and colorants in sunflower oil and spaghetti," Egyptian Journal of Agricultural Research, vol. 92, no. 1, pp. 309321, 2014.

[19] E. M. Rizk and K. H. Tolba, "Isolation and stabilization of carotenoids from sweet red pepper and utilization as food colorants," Arab Universities Journal of Agricultural Sciences, vol. 10, pp. 221-234, 2002.

[20] A. Debbouz, W. J. Pitz, W. R. Moore, and B. L. Dappolonia, "Effect of bleaching on durum-wheat and spaghetti quality," Cereal Chemistry, vol. 72, no. 1, pp. 128-131, 1995. 

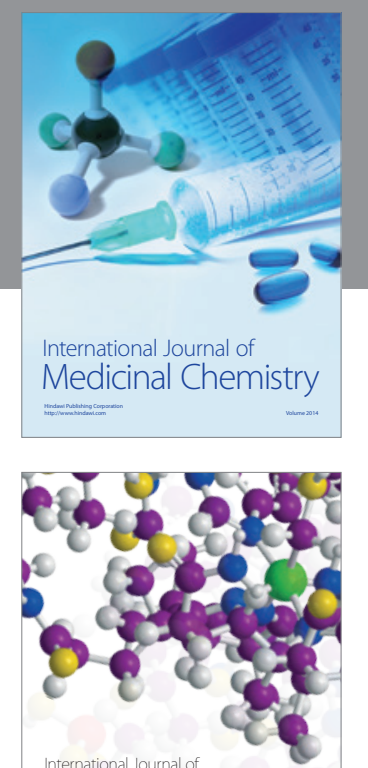

\section{Carbohydrate} Chemistry

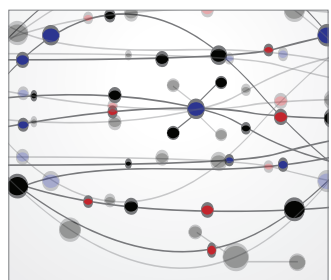

The Scientific World Journal
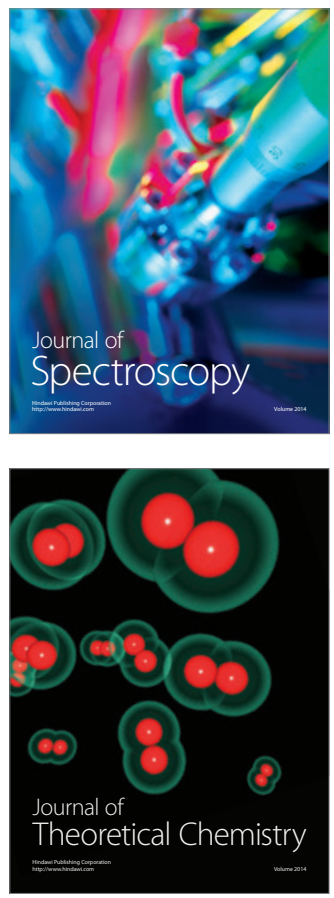
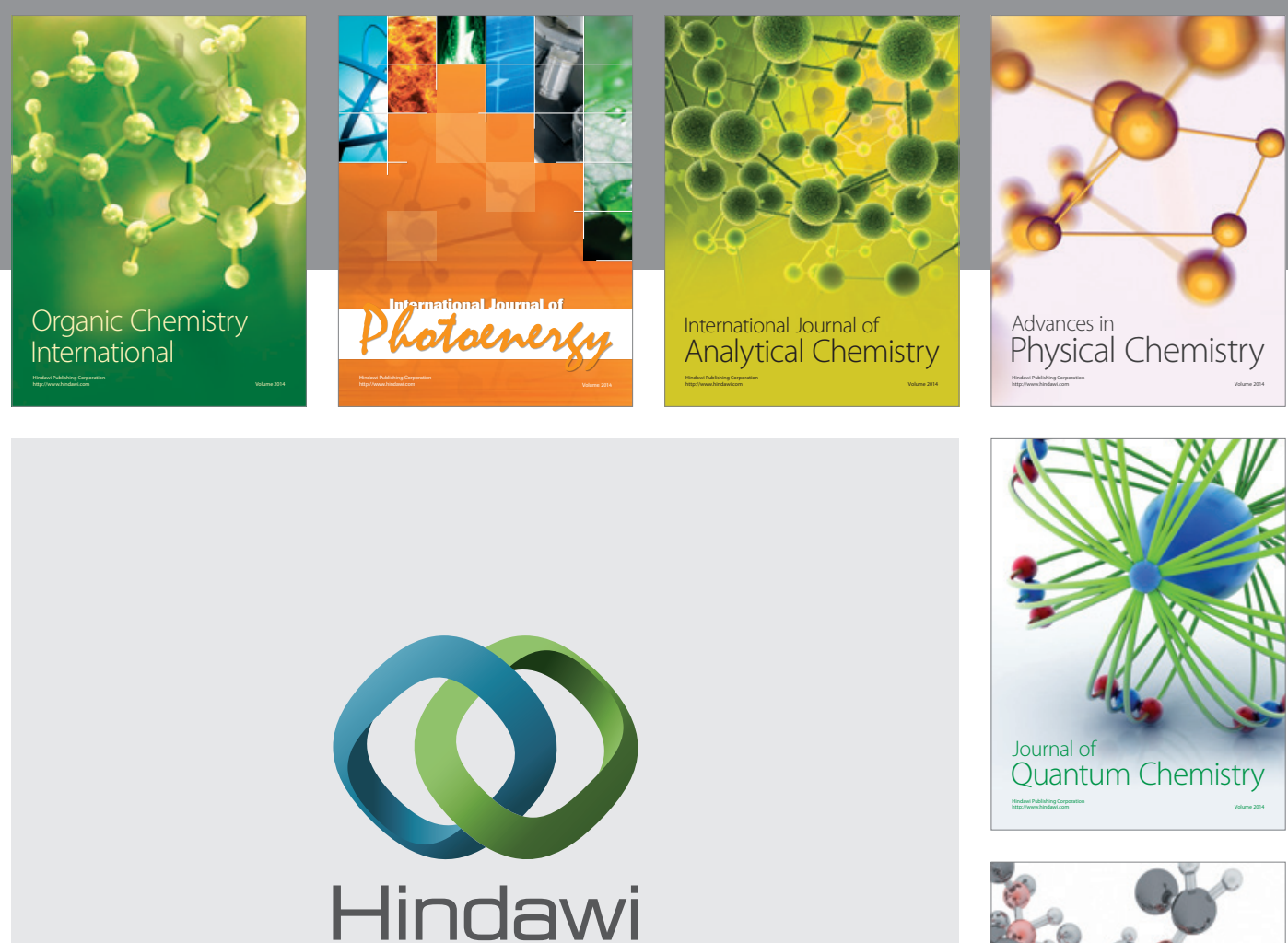

Submit your manuscripts at

http://www.hindawi.com

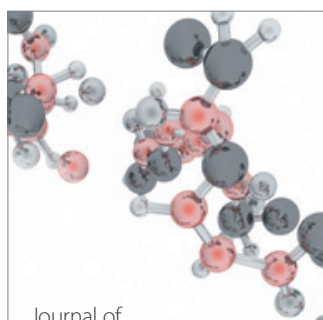

Analytical Methods

in Chemistry

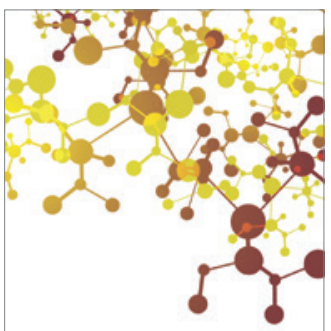

Journal of

Applied Chemistry

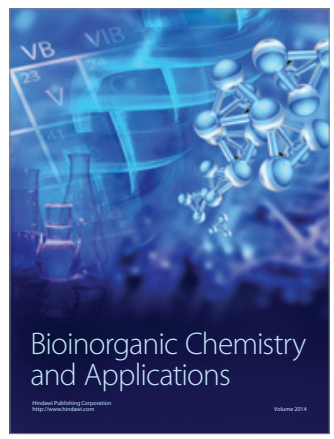

Inorganic Chemistry
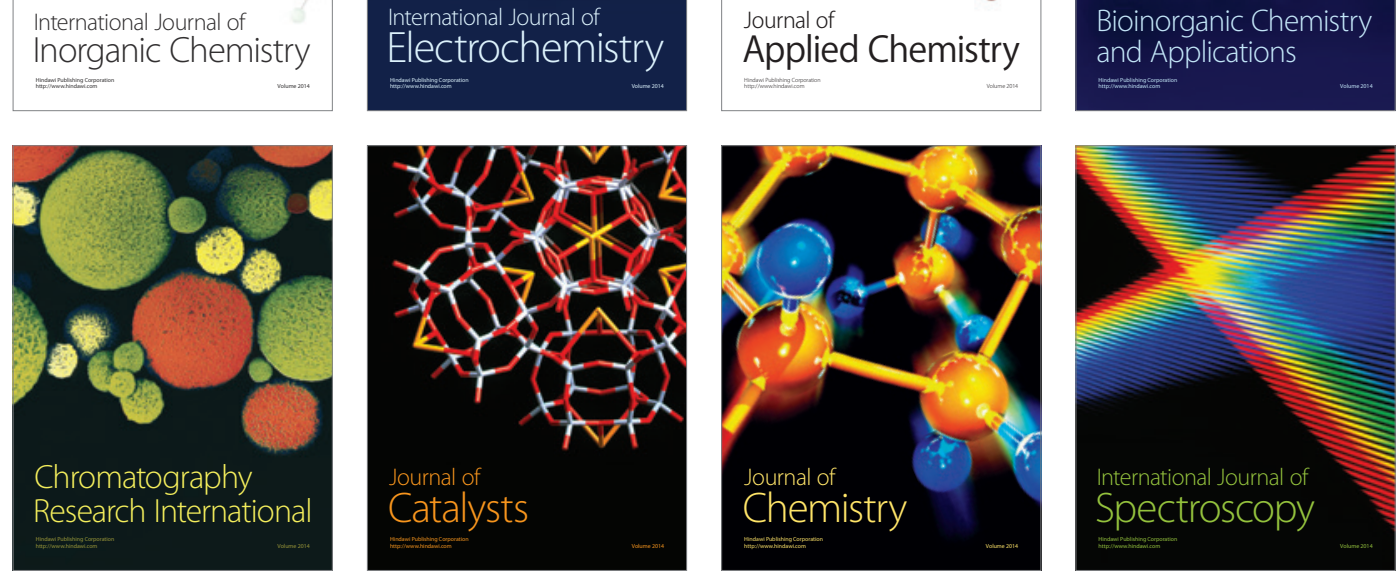